\title{
Correction: The dynamics of intonation: Categorical and continuous variation in an attractor-based model
}

\section{The PLOS ONE Staff}

\section{Notice of republication}

This article was republished on March 12, 2020, to correct formatting errors introduced during the typesetting process. The publisher apologizes for these errors. Please download this article again to view the correct version. The originally published, uncorrected article and the republished, corrected article are provided here for reference.

\section{Supporting information}

S1 File. Originally published, uncorrected article.

S2 File. Republished, corrected article.

\section{Reference}

1. Roessig S, Mücke D, Grice M (2019) The dynamics of intonation: Categorical and continuous variation in an attractor-based model. PLoS ONE 14(5): e0216859. https://doi.org/10.1371/journal.pone. 0216859 PMID: 31120891

\section{G OPEN ACCESS}

Citation: The PLOS ONEStaff (2020) Correction: The dynamics of intonation: Categorical and continuous variation in an attractor-based model. PLoS ONE 15(3): e0231221. https://doi.org/ 10.1371/journal.pone.0231221

Published: March 26, 2020

Copyright: ๑ 2020 The PLOS ONE Staff. This is an open access article distributed under the terms of the Creative Commons Attribution License, which permits unrestricted use, distribution, and reproduction in any medium, provided the original author and source are credited. 